increased risk for developing LCH. We presented 3 cases, 2 of whom aged 6 months and below.

Objectives To provide a wider perspective in diagnosing $\mathrm{LCH}$. Methods We are presenting 3 case reports, 2 of whom aged 6 months and below.

Results 1st case: Inguinal swelling mimicking inguinal abscess

This was a case report of a 6-month-old girl who presented with multiple lymphadenopathies over the right inguinal, axillary and cervical region. She was otherwise asymptomatic. A detailed history revealed presence of the right inguinal swelling noted since 2 weeks old and progressively increasing in size. Detailed investigation for infection were all normal. She received few courses of antibiotics but symptoms persisted. The father works in road construction industry. Excision biopsy of the right inguinal lymph node showed positive staining of CD1a, confirming the diagnosis of LCH.

2nd case: Skin rashes mimicking eczematous rashes

This was a 5-month-old girl who was admitted for fever with petechiae rashes, while the blood investigations revealed pancytopenia. 3 weeks prior to admission, she developed skin rashes mimicking eczema over the genitalia and bilateral inguinal. Systemic examinations showed hepatosplenomegaly, ascites with multiple petechiae rashes over the groin and neck region. Full blood picture reported pancytopenia with leucoerythroblastic blood film and circulating blasts. Her mother works in company selling household cleaning detergent. Skin biopsy of the inguinal region confirmed the diagnosis of $\mathrm{LCH}$.

3rd case: Scalp swelling mimicking scalp abscess

This was a 2-year-5-month old 'Orang Asli' (native people), whose stays in rubber estate in a remote area (regular exposure to pesticides), presented with multiple scalp swellings. Other systemic examination was unremarkable. Blood investigations and septic parameters were normal. Computed tomography (CT) brain showed multiple soft tissue mass with osteolytic bony destruction, involving outer and inner table of occipital, left parietal and left frontal bone. Excision biopsy of the scalp confirmed the diagnosis of LCH.

Conclusions LCH has a wide spectrum of clinical manifestations. Although it is a very rare disease, LCH should always be considered as differential diagnosis in children with generalised lymphadenopathy, scalp swelling or any disorder with multisystemic organs involvement. A thorough history to identify risk, physical examination and laboratory investigation are vital to aid in diagnosing patient.

\section{MATERNAL AND INFANT PREDICTORS OF GROWTH TRAJECTORIES IN SINGAPORE CHILDREN IN THE FIRST 18 MONTHS OF LIFE}

Tultul Das, Truls Ostbye, Yoke Hwee Chan, Ngiap Chuan Tan, Elaine Chew. Singapore

\subsection{6/bmjpo-2021-RCPCH.208}

Background Childhood obesity, with overweight defined as BMI $z$-score $>2$ based on WHO standards, is a growing problem in Singapore. The proportion of overweight children aged 6-18 years in Singapore has increased from 11\% in 2013 to $13 \%$ in 2017. This prevalence persists into childhood as $70 \%$ of children who were overweight when 7 years old remained overweight at 11 years of age. Rapid weight gain during infancy is an established risk factor for later obesity and excess body weight tracks from infancy into childhood and adulthood. However, little is known about the growth trajectories of infants in Singapore.

Objectives The objective of this study was to examine the longitudinal growth trajectories of infants in Singapore by tracking weight gain and Body Mass Index (BMI) (both absolute and BMI z-scores) in the first 18 months of life. Additionally, maternal and child predictors of infant weight and BMI were examined. Maternal predictors include number of months of breastfeeding, maternal age, and maternal education level while infant predictors include ethnicity and gender.

Methods Retrospective data on infants attending a government-subsidised primary care clinic between July 2019 to August 2020 was collected. Maternal characteristics (maternal age at birth and maternal education level at the child's 18month visit) and infant characteristics (birth weight, birth height, infant gender and infant ethnicity) were recorded from electronic medical records. Weight and BMI were measured at birth, 1, 3, 6, 9, 12 and 18 months. The type of feeding (breastfeeding or formula feeding or soft diet or a mix of any of the 3) was reported at each visit. Mixed Model Repeated Measures analysis was performed to compare weight and BMI within each gender, ethnicity and number of months of breastfeeding.

Results 7125 infants attended the clinic during the study period. $50.5 \%$ of the participants were male. The majority of infants were Chinese at $68.7 \%, 22.8 \%$ were Malay and $5.1 \%$ were Indian. We observed a peak in BMI at 6 months of age, with a mean of $17.5 \mathrm{~kg} / \mathrm{m}^{2}$. BMI then declined thereafter to $16.9 \mathrm{~kg} / \mathrm{m}^{2}$ at 12 months. We identified gender and ethnic differences in weight and BMI trajectories. Males had a higher mean weight and BMI than females across all time points. Chinese infants generally had a higher mean weight and BMI as compared to Malay and Indian infants. Indian infants exhibited the lowest BMI across all time points. With regards to feeding, $86.8 \%$ of the children were breastfed at 1 month of age and the prevalence declined to $54.8 \%$ by 6 months. Breastfeeding duration was identified as an important predictor of weight and BMI as a longer duration of breastfeeding was associated with a lower weight and BMI. Longer duration of breastfeeding was associated with a lower weight and BMI even when compared to those within the same gender and race.

Conclusions This study shows that gender, ethnicity and breastfeeding duration are early life predictors of rapid weight gain. Importantly, breastfeeding is a potentially modifiable predictor that may form the basis of targeted prevention efforts to reduce the risk of obesity.

\section{BONE DENSITY IN ASIAN ADOLESCENTS WITH ANOREXIA NERVOSA AND ATYPICAL ANOREXIA NERVOSA}

Eric Tay, Chu Shan Elaine Chew. Singapore

\subsection{6/bmjpo-2021-RCPCH.209}

Background Bone Mineral Density is frequently measured during the assessment and management of adolescents with Eating Disorders (EDs) such as Anorexia Nervosa (AN) and Atypical AN(AAN), which negatively affects the physical and mental developments of children at a vital phase of growth. Compromised bone accrual in adolescents has significant long-term implications on their peak bone mass and overall bone health 
in their later years. To date there has been no study on the effect of AN and AAN on BMD in young Asian adolescents.

Objectives In this retrospective study, we compare BMD and baseline characteristics in Adolescents from Singapore with AN and AAN, to find any difference in BMD Z-score between $\mathrm{AN}$ and AAN, and any predictive factors for low bone density in adolescents with EDs.

Methods We reviewed electronic medical records for all patients below the age of 18, who were treated for Eating Disorders at KK Women's and Children's Hospital between 2010 and $2020(\mathrm{n}=507)$.

Results 318 AN and 141 AAN cases were identified within the study period. Mean age of all cases is $14.08 \pm 1.49$ years with mean duration of illness being $8.06 \pm 6.15$ months. Females made up $92.8 \%$ of AN cases, compared to $84.4 \%$ of AAN cases $\left(\chi^{2}(1)=7.739 ; p=0.005\right) . \% \mathrm{mBMI}$ at presentation was significantly lower for AN than AAN (74.55 \pm 7.27 versus $97.12 \pm 9.95, t(210)=-24.18, p<0.001) .29 \%$ of AAN patients were premorbidly overweight $(\max$ IBW $>120 \%)$, compared to $4.6 \%$ of AN patients $\left(\chi^{2}(1)=42.97 ; p<\right.$ 0.001). There were significantly higher BMD Z-scores in AAN compared to AN for both the spine $(1.55 \pm 1.63$ versus 0.29 $\pm 1.67, \mathrm{t}(179)=-4.22, \mathrm{p}<0.001)$ and femur $(0.48 \pm 0.93$ versus $0.23 \pm 1.19, \mathrm{t}(194)=-3.63, \mathrm{p}<0.001)$ region. In our linear regression model, BMD Z-score was not associated with duration or magnitude of weight loss as well as duration of amenorrhea or overweight status.

Conclusions From the findings of our study, we conclude that asian adolescents with AAN demonstrated higher BMD Zscores for both spine and femur compared to their AN counterparts. There was no predictive factors for low bone density. These findings may be instructive in informing guidelines for the management of Asian adolescents with AN and AAN.

\section{INTRAVENTRICULAR HAEMORRHAGE IN A UGANDAN COHORT OF LOW BIRTH WEIGHT NEONATES: THE IVHU STUDY}

Rachael MacLeod, Noella Okalany, Leigh E Dyet, Cornelia F Hagmann, Frances M Cowan, Kathy Burgoine. UK

\subsection{6/bmjpo-2021-RCPCH.210}

Background Globally, 15 million neonates are born prematurely every year, over half in low income countries (LICs). Premature and low birth weight neonates have a higher risk of intraventricular haemorrhage (IVH). There are minimal data regarding IVH in sub-Saharan Africa. This is one of the first studies of IVH in LBW neonates in a LIC in sub-Saharan Africa and the first in east Africa.

Objectives The objective of this study is to examine the incidence, severity and timing of and modifiable risk factors for IVH amongst low-birth-weight neonates in Uganda.

Methods This is a prospective cohort study of neonates with birthweights of $\leq 2000 \mathrm{~g}$ admitted to a neonatal unit (NU) in a regional referral hospital in eastern Uganda. Maternal data were collected from interviews and medical records. Neonates had cranial ultrasound (cUS) scans on the day of recruitment and days 3, 7 and 28 after birth. Risk factors were tabulated and are presented alongside odds ratios (ORs) and adjusted odds ratios (aORs) for IVH incidence. Outcomes included incidence, timing and severity of IVH and 28-day survival.
Results Overall, 120 neonates were recruited. IVH was reported in 34.2\% of neonates; $19.2 \%$ had low grade (Papile grades 1-2) and $15 \%$ had high grade (Papile grades $3-4$ ). Almost all IVH (90.2\%) occurred by day 7, including $88.9 \%$ of high grade IVH. Of those with known outcomes, $70.4 \%$ $(81 / 115)$ were alive on day 28 and survival was not associated with IVH.

We found that vaginal delivery, gestational age (GA) $<32$ weeks and resuscitation in the NU increased the odds of IVH. The aOR for having any IVH was 3.5 (95\% CI 1.0116.45), comparing vaginal delivery with Caesarean delivery. Compared with neonates of $\geq 32$ weeks GA, neonates of GA $<32$ weeks had increased odds of any IVH, aOR $6.70(95 \%$ CI 1.6-31.02), high grade IVH, aOR 8.18 (95\% CI 1.1869.37), and low grade IVH, aOR 6.70 (95\% CI 1.12-46.9). Neonates who required resuscitation in the $\mathrm{NU}$ also had increased odds of any IVH, aOR 5.10 (95\% CI 1.23-26.36) and high grade IVH aOR 9.24 (95\% CI 1.83-54.38). Neonates who were SGA (small for gestational age, <10th centile) had increased odds of low grade IVH, aOR 9.96 (95\% CI 1.83-71.84). Of the 6 neonates who received 2 doses of antenatal steroids, none had IVH.

Conclusions This study found that in this resource limited NU in a regional referral hospital in eastern Uganda, more than a third of neonates born weighing $\leq 2000 \mathrm{~g}$ had an IVH and majority of these occurred by day 7 . We found that vaginal delivery, GA < 32 weeks, resuscitation in the NU and being SGA were associated with increased odds of having an IVH. This study had a high rate of SGA neonates and the risk factors and relationship of these with IVH in this setting needs further investigation. The role of antenatal steroids in the prevention of IVH in LICs also needs urgent exploration.

\section{THE UTILITY OF C-REACTIVE PROTEIN FOR SCREENING AND DIAGNOSIS OF EARLY-ONSET INFECTION IN TERM NEONATES}

Xuelian Wang, Hugh Simon Lam. China

10.1136/bmjpo-2021-RCPCH.211

Background Early onset sepsis (EOS) remains a significant cause of neonatal mortality and morbidity. About $10 \%$ of term neonates were exposed to antibiotics for EOS risk, despite a very low incidence (around 1\%o) in this group especially after the introduction of intrapartum antibiotic prophylaxis. One of the main reasons for over treating the neonates is the lack of ideal diagnostic biomarkers. C-reactive protein (CRP) and complete blood count (CBC) are most commonly used biomarkers for EOS, but the diagnostic performance of CRP was poorly understood with most studies on late onset sepsis. In the past 15 years, our neonatal unit has used CRP systematically in neonates with risk or signs of EOS, providing a good opportunity to analyze its diagnostic accuracy.

Objectives In this study, we aimed to evaluate the performance of CRP for early onset infection diagnosis in symptomatic neonates and screening in asymptomatic high-risk neonates.

Methods This is a retrospective study done in a tertiary neonatal care center in Hong Kong. Term neonates born during the period of 01/01/2005-30/06/2018 with blood taken for CRP testing in the first 72 hours were included. Their CRP results were included into the analysis if blood were taken before antibiotic treatment. Subjects were divided into four 